\title{
Impact of a liquid drop on a granular medium: Inertia, viscosity and surface tension effects on the drop deformation
}

\author{
E. Nefzaoui ${ }^{a}$, O. Skurtys ${ }^{b, *}$ \\ a Institut P', CNRS-Université de Poitiers-ESIP, Bâtiment de Mécanique, 40, Av. du Recteur Pineau, 86022 Poitiers Cedex, France \\ ${ }^{\mathrm{b}}$ Department of Mechanical Engineering, Universidad Técnica Federico Santa María, Av. Vicuña Mackenna 3939, Santiago, Chile
}

\section{A R T I C L E I N F O}

\section{Article history:}

Received 2 January 2011

Received in revised form 11 February 2012

Accepted 10 March 2012

Available online 29 March 2012

\section{Keywords:}

Crater morphology

Drop impact

Granular media

Drop spreading

\begin{abstract}
A B S T R A C T
An experimental study of liquid drop impacts on a granular medium (glass beads) is proposed. Four fluids were used to vary physical properties: pure distilled water, water with glycerol at two concentrations $1: 1$ and $1: 2 \mathrm{v} / \mathrm{v}$ and water with surfactant (Tween 20 ) at the concentration of $0.1 \mathrm{~g} \mathrm{l}^{-1}(\approx 1 \mathrm{CMC})$. The drop free fall height was varied to obtain a Weber number (We) between 10 and 2000 and a Reynolds number (Re) between 100 and 13000. Different behaviors during the drop spreading, receding and absorption are highlighted as function of the fluids viscosity $(\mu)$ and surface tension $(\gamma)$. The role plays by the surfactant on the drop behavior after impact was found significant especially when the kinetic energy was high. On the other hand, the final diameter of the drop was found insensitive to the viscosity of the fluid and the Weber number. The maximal spreading factor $\beta_{\max }=D_{\max } / D_{0}$ was found to scale as $W e^{\frac{1}{4}}$ for water + surfactant drops $\left(\gamma \approx 36 \mathrm{~mJ} \mathrm{~m}^{-2}, \mu=1 \mathrm{mPa}\right.$ ) whereas for the other fluids $\left(\gamma \approx 70 \mathrm{~mJ} \mathrm{~m}^{-2}\right.$ but $\mu=1-19 \mathrm{mPa}$ s) $\beta_{\max } \sim W e^{\frac{1}{5}}$. The boundary between splashing and non-splashing was determined using the splash parameter $K_{d}=W e^{1 / 2} \mathrm{Re}^{1 / 4}$. The threshold value $K_{d s}$ was found higher that for impact of liquid drops on solid dry substrates. Experimental absorption times are also commented and compared with a simplified theoretical model. Finally, the morphology of the craters is discussed and a relationship between the crater diameter and the drop kinetic energy at impact is presented.
\end{abstract}

(c) 2012 Elsevier Inc. All rights reserved.

\section{Introduction}

The phenomenon of droplet impact on non-cohesive granular media is present in various industrial processes as food engineering, candy coated pills fabrication, starch wetting, etc. [1]. It is also of great importance in environmental sciences: water erosion of soil, pollution of rivers and lakes, rainfall simulations, etc. [2,3]. However, studies of this phenomenon are very scarce. Holman et al. [4] studied the micro-metric drop impact on micro-metric grains of the same size. Besides, authors used a very particular fluid, an aqueous polymer solution in this case. Others studied drops imbibition into powder beds with no impact. The drops were deposited on the granular surface with no kinetic energy [5]. Recently, the problem has been investigated by Katsuragi [6] where the author only focused on the granular medium deformation, in particular the resulting craters morphologies for different water drop velocities and granular media (grains sized between 4 and $50 \mu \mathrm{m}$ ). The existence of different crater shapes was reported depending on the drop free fall height. Moreover, the drop maximal extensions, $D_{\max }$, assumed equal to the crater diameter,

\footnotetext{
* Corresponding author. Tel.: +56 24326729.

E-mail addresses: nefzaoui.elyes@univ-poitiers.fr (E. Nefzaoui),olivier.skurtys@ usach.cl (O. Skurtys)
}

was scaled as $W e^{1 / 4}$. However, Nefzaoui and Skurtys [7] reported that, for water drops impact on coarser grains (average particle diameter $\left.D_{g} \sim 81 \mu \mathrm{m}\right), D_{\max } \sim W e^{1 / 5}$ and that the crater diameter, $D_{\text {rim }}$, was not equal to $D_{\max }$.

On the other side, the impact of a liquid drop on the solid surface has been thoroughly studied for over a century [8]. Even if the drop behavior after collision with the solid surface is very complex since it depends on the physico-chemical characteristics of the drop and the impact surface, the impact phenomenon can be divided in several sub-processes identified as spreading, receding, splashing and bouncing [8-11]. Recently, studies have been focused on the role of the roughness and texture of a solid surface on the drop impact process [12,13]. In particular, it was shown that micro-grooves influence both the spreading and receding processes of an impacting drop [12].

Spherical solid impact into granular media were investigated to some extent decades ago [14-18]. For impact energies ranged between $10^{-2}$ and $0.6 \mathrm{~J}$, the morphology of the craters was reported in detail by Walsh et al. [16]. In particular, the scaling of crater dimensions has been studied, and a power-law relationship between crater diameter $D_{c}$ and the energy of impact $E_{K}$ can be derived in certain limits: $D_{c} \propto E^{\frac{1}{4}}$. The process of crater formation is complex since the resistance of the granular medium to the projectile penetration depends on the medium intrinsic properties, 
Table 1

Measured physical properties of fluids, the drop diameter $\left(D_{0}\right)$ and the Ohnesorge number $\left(\right.$ Oh $\left.=\frac{\mu}{\sqrt{\rho \gamma D_{0}}}\right)$

\begin{tabular}{|c|c|c|c|c|c|}
\hline Fluid & $\rho\left(\mathrm{kg} \mathrm{m}^{-3}\right)$ & $\mu(\mathrm{mPa} \mathrm{s})$ & $\gamma\left(\mathrm{mJ} \mathrm{m}^{-2}\right)$ & $D_{0}(\mathrm{~mm})$ & $\mathrm{Oh} \times 10^{3}$ \\
\hline Water & 996 & 1 & 72.2 & $2.88-3.25-3.65$ & $1.95-2.2$ \\
\hline Water-glycerol 1:1 & 1121 & 6 & 66.9 & 3.47 & 11.8 \\
\hline Water-glycerol $1: 2$ & 1162 & 19 & 65.1 & 3.39 & 37.5 \\
\hline Water-Tween $20\left(0.1 \mathrm{~g} \mathrm{l}^{-1}\right)$ & 996 & 1 & 36 & 3 & 3 \\
\hline
\end{tabular}

its packing mode and the applied force. Indeed, impacting sphere penetration dynamics and grain ejection were observed to be very different whether the granular material is loose or dense [18]. Another variant of these experiments concerns a bead impact on a granular target made of similar beads [19].

In this paper, an experimental study of drop impacts on a granular medium (glass beads) is proposed. Four different liquids were used in order to study the effect of viscosity and surface tension on the drop deformation for different impact velocities and try to determine the relevant parameters that govern the phenomenon. The impact dynamics are described through the temporal evolution of the spreading factor using a high speed video. Different behaviors are highlighted depending on the receding magnitude and occurrence of splashing. A power law for the maximal spreading drop diameters dependence on the Weber number is proposed. Moreover, a relationship delimiting the boundary between splashno splash is determined. Experimental absorption times are also commented and compared with a simplified theoretical model. Final, crater morphologies obtained by varying the drop kinetic energy at impact over more than three orders of magnitude are presented and discussed.

\section{Material and methods}

\subsection{Physical properties of liquids}

Four fluids were used: pure distilled water, water with glycerol at 2 concentrations $1: 1$ and $1: 2 \mathrm{v} / \mathrm{v}$ and water with a surfactant (Tween 20 ) at the concentration of $0.1 \mathrm{~g} \mathrm{l}^{-1}(\approx 1 \mathrm{CMC})$. Their physical properties were measured and are presented in Table 1.

Interfacial tension measurements were carried out by the pendant drop method [20]. A small drop of liquid (about $10-20 \mu \mathrm{l}$ ) attached to the tip of a stainless-steel needle (inner diameter of $1.651 \pm 0.005 \mathrm{~mm}$ ) was suspended into air under a constant temperature $\left(\sim 21^{\circ} \mathrm{C}\right)$. The drop was formed by a controlled syringe pump (Model 1000, New Era Pump System Inc., Farmingdale, NY, USA). Images of the drop were taken with the CCD camera equipped with a zoom objective. The shape of the drop at equilibrium, determined by the balance of gravity and interfacial tension, was determined from the fundamental Laplace equation:

$\frac{d \sin \theta}{d x}=\frac{2}{b}-\frac{g \Delta \rho}{\gamma} z-\frac{\sin \theta}{x}$

where the origin of the coordinate system was at the drop apex; $x$ and $z$ were the Cartesian coordinates at any point of the droplet, $b$ was the radius curvature at the apex; $\Delta \rho$ was the difference between both densities; $\theta$ is the angle between the drop axis and the normal to the drop interface. The solution of Eq. (1) was obtained with the free software Octave. To validate the results it was corroborated experimentally that the interfacial tension of the pure water/air system was $72.2 \pm 0.3 \mathrm{~mJ} \mathrm{~m}^{-2}$. Finally, the rheological properties of each liquid were measured by a rheometer (Carri-Med model CS 100 , Germany) at room temperature $\left(\sim 21^{\circ} \mathrm{C}\right)$.

\subsection{Apparatus for drop impact}

The apparatus consisted of a drop production system and an image acquisition system. Liquid drops were generated by a precision flat tipped syringe needle (Sigma-Aldrich, USA, St. Louis) connected to a digitally controlled syringe pump (Model 1000, New Era Pump System Inc., Farmingdale, NY, USA). Liquid flow rate was sufficiently low to get a nil drop initial velocity $\left(0.05 \mathrm{ml} \mathrm{min}^{-1}\right)$. Three flat tipped needles (gauge $16,18,20$ ) were used to obtain various drop diameters $D_{0}$, between 2.88 and $3.65 \mathrm{~mm}$.

A high-speed video camera (Pulnix model TM-6740GE, San Jose, CA, USA) with a zoom video lens $(18-108 \mathrm{~mm}$ f/2.5D, Edmund Optics, NJ, USA) was used to capture the drop impact and the spreading process. Camera was mounted on a boom stand and could capture 200 frames per second (fps) in full frame $(640 \times 480)$ and $1250 \mathrm{fps}$ with a reduced matrix resolution $(224 \times 160)$.

Drops are assumed with some assurance to maintain a spherical shape through the free fall since their radius were lower than the capillary length, $\kappa=\sqrt{\frac{\gamma}{\rho g}}$ [21]. Drop impact velocities $V_{0}$ ranged from 0.4 to $5 \mathrm{~m} \mathrm{~s}^{-1}$ and were increased or decreased by changing the drop release height $h(10<h<1300 \mathrm{~mm})$. For each impact, $V_{0}$ was measured from captured images. The kinetic energy of the drop at impact, given by $E_{K}=\frac{\pi}{6} \rho D_{0}^{3} g h$ was varied between $1 \times 10^{-6}$ and $3 \times 10^{-4} \mathrm{~J}$ whereas surface energy of the free drop surface, $E_{S}=\pi D_{0}^{2} \gamma$ was varied between $1.8 \times 10^{-6}$ and $3 \times 10^{-6} \mathrm{~J}$.

\subsection{Granular medium and impact surface preparation}

The granular medium was a commercial dry glass beads (G8893, Sigma-Aldrich, USA, St. Louis) of density $\rho_{\mathrm{g}} \approx 2300 \mathrm{~kg} \mathrm{~m}^{-3}$ with a measured mean diameter $D_{g} \sim 81 \mu \mathrm{m}$. Ninety percent of the mass fraction was comprised between $53 \mu \mathrm{m}$ and $90 \mu \mathrm{m}$. This granular medium was chosen sufficiently coarse to suppose that cohesive force (like the van der Waals force) may be considered negligible. Indeed, van der Waals force is important for granular medium with particle diameters close to $10 \mu \mathrm{m}$ [22]. However, the granular medium was thin enough so that its surface is deformed by the impact. A circular container of $78 \mathrm{~mm}$ in diameter and $14 \mathrm{~mm}$ in height was filled. We observed that the initial state of the granular medium had a great effect on the crater formation therefore it was important to keep the same initial state of the granular bed in general, and of the compaction in particular. For this purpose, the same protocol was respected for each sample preparation. Beads were gently rolled into the container using a glass funnel. The container was overfilled and the surface was leveled using a straight-edge to produce an almost uniform surface. Finally, the container was weighed to check that the mass of beads was identical for each experiment. The packing fraction was measured by determining the ratio between the volume of the glass beads and the volume of the loose structure made by the glass beads [23]. The volume of the glass beads $\left(V_{g}\right)$ was determined from their total mass $\left(M_{g}\right)$ and density $\left(\rho_{g}\right): V_{g}=M_{g} / \rho_{g}$. Packing fraction (or bulk density) of about $0.60 \pm 0.02$ was maintained constant for each experiment. 


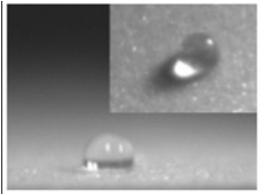

$t \approx 0 \mathrm{~ms}$

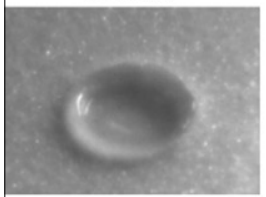

$t=2.4 \mathrm{~ms}$

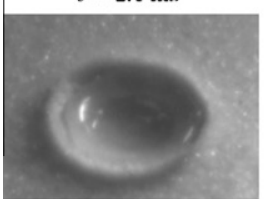

$t=4.8 \mathrm{~ms}$

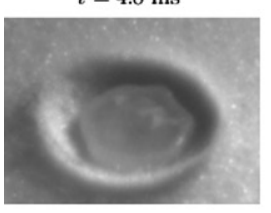

$t=8 \mathrm{~ms}$

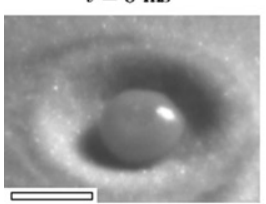

$t=20 \mathrm{~ms}$

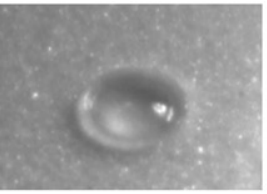

$t=0.8 \mathrm{~ms}$

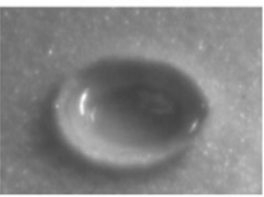

$t=4 \mathrm{~ms}$

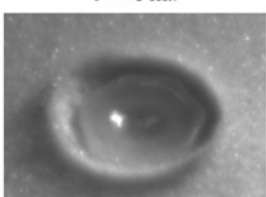

$t=6.4 \mathrm{~ms}$

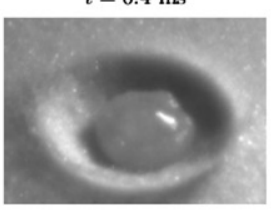

$t=11.2 \mathrm{~ms}$

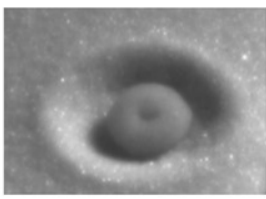

$t=128 \mathrm{~ms}$

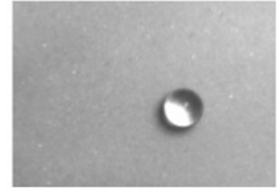

$t \approx 0 \mathrm{~ms}$

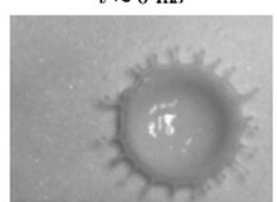

$t=2.4 \mathrm{~ms}$

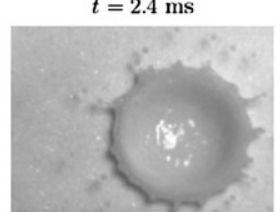

$t=4.8 \mathrm{~ms}$

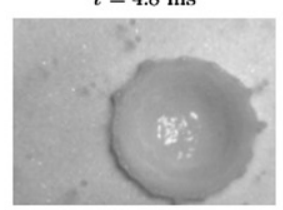

$t=8 \mathrm{~ms}$

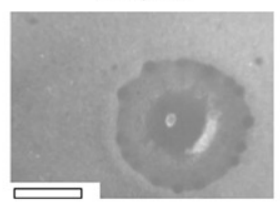

$t=20 \mathrm{~ms}$

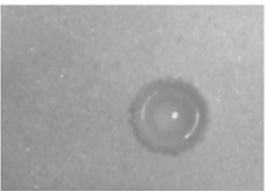

$t=0.8 \mathrm{~ms}$

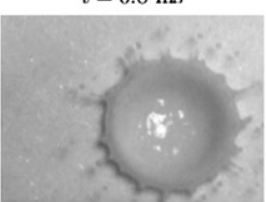

$t=4 \mathrm{~ms}$

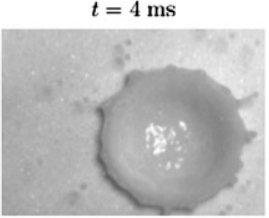

$t=6.4 \mathrm{~ms}$

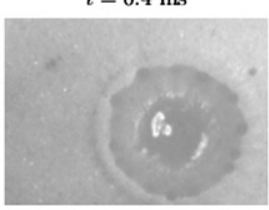

$t=11.2 \mathrm{~ms}$

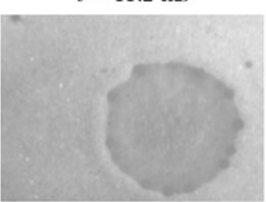

$t \gtrsim 90 \mathrm{~ms}$

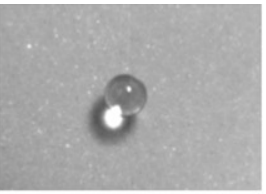

$t \approx 0 \mathrm{~ms}$

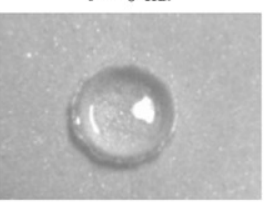

$t=1.6 \mathrm{~ms}$

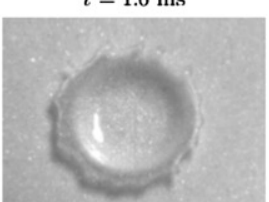

$t=3.2 \mathrm{~ms}$

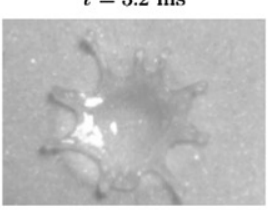

$t=12 \mathrm{~ms}$

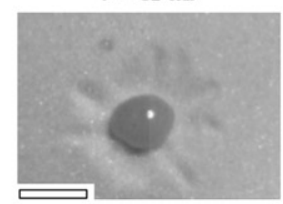

$t=65.6 \mathrm{~ms}$

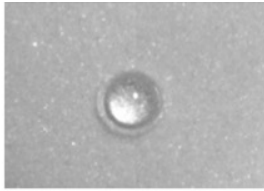

$t=0.8 \mathrm{~ms}$

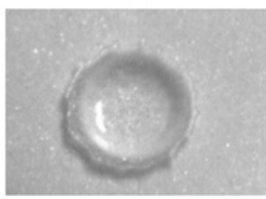

$t=2.4 \mathrm{~ms}$

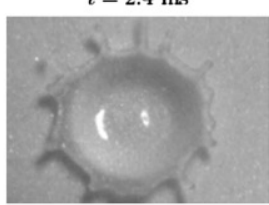

$t=6.4 \mathrm{~ms}$

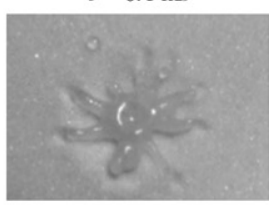

$t=17.6 \mathrm{~ms}$

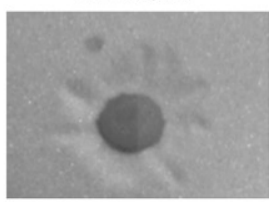

$t \gtrsim 1248 \mathrm{~ms}$

(A)

(B)

(C)

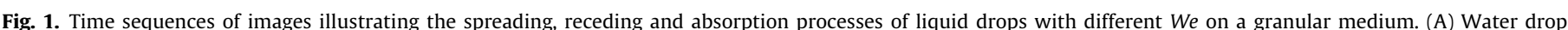

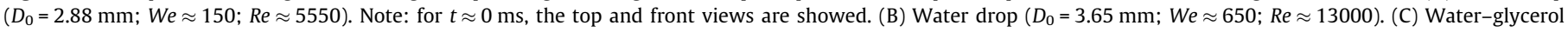
mixture (bulk ratio $1: 1)\left(D_{0}=3.47 \mathrm{~mm}\right.$; $\left.W e \approx 570 ; R e \approx 2000\right)$. Each bar represents: $5 \mathrm{~mm}$.

\subsection{Fluid-granular contact angle measurements}

The contact angle of water over the granular media was measured through a capillary suction experiment as detailed by Xue et al. [24]. The liquid front height $(z)$ of the fluid rising in a granular column (due to capillary forces) was measured as function the time $(t)$. Taking into account hydrostatic, viscous and capillary effects (inertial effects were neglected), a relation between the contact angle and the other measured physical parameters can be obtained [24]:

$\gamma^{2} \cos ^{2}\left(\theta_{d}\right)=\frac{2 \rho g \mu}{3} \frac{z^{3} v}{z-2 t v}$

where $\gamma, \rho, \mu$ and $\theta_{d}$ are the fluid surface tension, density, dynamic viscosity and the fluid-granular advancing contact angle respectively. From, $t$ and $z$ the fluid front velocity $v$ was calculated: $v=\frac{d z}{d t}$. Thus, the only unknown $\theta_{d}$ was easily deduced from Eq. (2) since other parameters were measured experimentally.

\subsection{Granular medium pore diameter measurements}

An estimation of the mean pore radius of the granular medium was needed to determine the drop absorption times. As reported by Xue et al. [24], if the hydrostatic effects are very significant, the mean pore radius, $R_{p}$, follows the relationship:

$R_{p}=\frac{z^{2}}{\gamma \cos \left(\theta_{d}\right)} \cdot \frac{1}{\frac{t}{2 \eta}-\frac{\rho g z^{3}}{3 \gamma^{2} \cos ^{2}\left(\theta_{d}\right)}}$ where $z$ is liquid front height, $t$ the time, $\gamma$ the fluid surface tension, $\rho$ the fluid density, $\mu$ the dynamic viscosity and $\theta_{d}$ the fluid-granular advancing contact angle. This relation leads to consistent results when inertial effects are negligible, i.e. not at early times when $t$ and $z$ are too small.

\subsection{Drop impact measurements}

For all video data, initial drop diameter $\left(D_{0}\right)$ and instantaneous spreading diameter $(D(t))$ were measured using an image analysis software (ImageJ, National Institutes of Health, USA). $D(t)$ was accurately calculated using the Feret's diameter: $D_{i}(t)=2 \sqrt{A_{i}(t) / \pi}$ where the subscript $i$ is [max, end, rim] and $A_{i}$ is the measured drop's (or crater's) area. From these measurements, the temporal evolution of the spreading factor, the maximum diameter $\left(D_{\max }\right)$, the final diameter $\left(D_{\text {end }}\right)$ and the crater rim diameter $\left(D_{\text {rim }}\right)$ were deduced. Finally, the absorption time of the drop by the granular medium $\left(t_{\text {measured }}\right)$ after impact was also measured from an inspection of the video data. Absorption was considered to be achieved when no visible difference between two and more consecutive frames was observed.

\section{Results and discussion}

A mathematical formulation of the impact leads to consider the continuity and momentum equations in the radial and axial directions. Three dimensionless numbers can be deduced after the 
dimensional analysis: Reynolds $\left(R e=\frac{\rho V_{0} D_{0}}{\mu}\right)$, Weber $\left(W e=\frac{\rho D V_{0}^{2}}{\gamma}\right)$, Froude $\left(F r=\frac{V_{0}^{2}}{g D}\right)$. As in all experimental measurements, the gravitational effect was negligible compared to inertia $\left(\mathrm{Fr}^{-1} \ll 1\right)$. only the Weber number, which measures the relative importance of kinetic and surface energy of the drop, and the Reynolds number $(R e)$, ratio of inertial to viscous forces were retained as parameters of the problem. Thus, the impact drop dynamics was driven by an interplay between the kinetic energy, viscosity and the droplet surface tension. Another dimensionless number, the Ohnesorge number $\left(O h=\frac{\mu}{\sqrt{\rho \gamma D_{0}}}=\frac{\sqrt{W e}}{R e}\right)$, can be used to take simultaneously into account the three parameters [8]. The three typical phases observed after the drop impact on the granular medium, i.e. the spreading, receding and absorption, are detailed in the next paragraphs.

\subsection{Drop dynamics}

In all experiences, the drop's kinetic energy at impact was sufficient to spread, $W e>8$. Fig. 1 shows time sequences of images of drop impact on a granular medium for different We and fluids. From an inspection of all video data, the fluid motion after impact on the granular medium can be divided in three phases: spreading, receding and absorption. As in the case of the drop impact on solid porous substrate, drop absorption seemed to be the last fluid motion stage [25]. In order to describe with more details and accuracy the impact phenomenon, the temporal evolution of the spreading factor $\beta(t)$ after impact is presented in Fig. 2. In the usual way, for inertia governed impacts, time $t$ was made dimensionless using the impact velocity $V_{0}$ and the initial spherical drop diameter $D_{0}$.

\subsubsection{Spreading stage}

Spreading $\left(\frac{d \beta}{d t^{*}}>0\right)$ was the first stage of drop deformation after impact. The drop was initially flattened and spread out horizontally into a pancake shape. Even if the description of the drop deformation before $t=0.8 \mathrm{~ms}$ was beyond our experimental capacities, it was observed that in the first instants, the drop was not deformed (keep a spherical shape). It may be supposed that the drop has penetrated into the porous granular medium (see in Fig. $1 \mathrm{~A}$ for $t \approx 0 \mathrm{~ms}$ ). Indeed, to the difference of the solid surface where the drop deformation starts at impact time (the drop diameter evolution scales as $\left.t^{\frac{1}{2}}[9]\right)$, the granular surface was porous and can be deformed during the impact. The drop spread to its maximum value, $\beta_{\max }$, in a very short time, $t^{*}<5$.

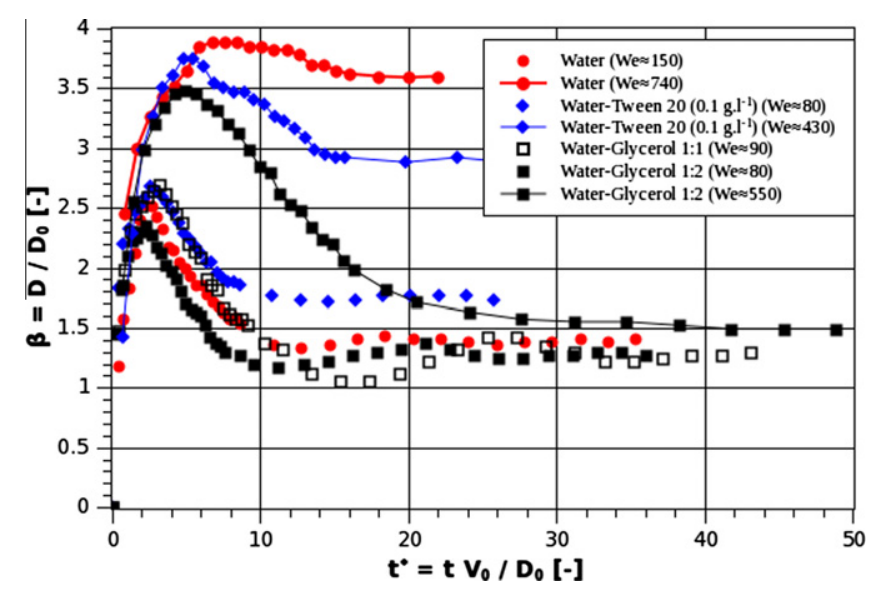

Fig. 2. Temporal evolution of the spreading factor, $\beta(t)=D(t) / D_{0}$, for different Weber numbers and fluids.
When Tween 20 was added to water, it was interestingly to observe in Fig. 2 that $\beta_{\max }$ (water + Tween $)>\beta_{\max }$ (water) for the same We number. Indeed, if $\beta_{\max }$ (water + Tween $) \approx \beta_{\max }$ (water) then $W e_{\text {water }}$ was around $40 \%$ higher than $W e_{\text {water+Tween }}$. This may be attributed to the reduction in surface tension at both the liquidvapor and liquid-granular interfaces due to the adsorption of the surfactant molecules. However, it is well-known that in the case of the drop impact where the free surface area grows rapidly ( $<5 \mathrm{~ms}$ ), very complicated dynamic effects can occur, for example, the surfactant can take much larger time to disperse and adsorb at the interface and equilibrium conditions are not reached [26].

The fluid viscosity was increased by adding glycerol to water of about 1-19 mPa s. In all case, the surface tension was maintained close to that of water. As seen in Fig. 2, the viscosity slowed the spreading since $\beta\left(t^{*}\right)$ (water + glycerol $\left.1: 2\right)<\beta\left(t^{*}\right)$ (water + glycerol $1: 1)$. This phenomenon may be explained by the increasing of the viscous dissipation.

\subsubsection{Receding stage}

Receding $\left(\frac{d \beta}{d t^{*}}<0\right)$ was the second stage of drop deformation after impact (see Fig. 2). When the surface tension was decreased of about $72.2 \mathrm{~mJ} \mathrm{~m}^{-2}$ (water) to $36 \mathrm{~mJ} \mathrm{~m}^{-2}$ (water + Tween), the behavior of the receding was considerably different at intermediate (and at low We all the more) and high $W e$. For $W e_{\text {water }} \approx 150$, a "total receding" was observed, the drop final spreading factor is relatively small: $D_{\text {end }} \simeq 1.4 D_{0}$ (water) and $D_{\text {end }} \simeq 1.7 D_{0}$ (water + Tween). Besides, when it reached its final diameter, the fluid recovered a drop-like shape (see Fig. 1A, for $t>20 \mathrm{~ms}$ ). Actually, at low and intermediate $W e$, the drop spherical shape recovery let think that receding was governed by capillary forces. When the kinetic energy of drop was increased, $W e_{\text {water }} \approx 740$ and $W e_{\text {water+Tween }} \approx 430$ (Tween), the final drop diameter was found closer to $\beta_{\max }$ : $D_{\text {end }} \simeq 3.5 D_{0}$ (water) and $D_{\text {end }} \simeq 2.9 D_{0}$ (water + Tween). In contrast with the previous situation, we can talk about "partial receding" (see Fig. 1, for $t>8 \mathrm{~ms}$ ). In this case, the kinetic energy of the spreading has dominated the drop surface energy and various fingers could be observed along the outer rim of the droplet. Furthermore, if the kinetic energy was sufficiently high to overcome the surface energy available in the fingers, the fingers could be pinched off into smaller droplets and the drop was splashed.

When the fluid viscosity was the modified parameter, Fig. 2 shows that the drop diameter receded to the final drop diameter $\beta_{\text {end }} \simeq 1.3$. This value was found smaller than $\beta_{\text {end }}$ (water + Tween $)$. Indeed, unlike to the previous case, where the surface tension was the modified physical property, no significant difference on the final drop diameter (after receding) was observed between high and low We impacts and between low and high viscosity. However, the increasing viscosity produced a decreasing in $D_{\max }$. Furthermore, after the receding process, oscillations of the drop diameter were observed for low We.

\subsubsection{Maximum drop deformation as function of We and Re}

For a large Weber number range (10-2000) and for the four considered liquids, the maximum spread factor $\left(\beta_{\max }=D_{\max } / D_{0}\right)$ of drops impacting on the granular medium is presented in Fig. 3A. For all liquids, the values of $\beta_{\max }$ increased with We due to the increase of drop impact velocity. Thus, a large We number means that the drop spreads to a maximum radius much greater than its initial value, acquiring a large surface area. This result allows to conclude that the spreading stage is controlled by the inertia in the same way that the drop impact on a solid surface as reported by Sikalo et al. [27].

For water + Tween drops, i.e. for low surface tension, the maximum spreading factor was well fitted by a straight line of slope $0.24 \pm 0.01$ (with a high coefficient of determination, $R^{2}=0.99$ ). 


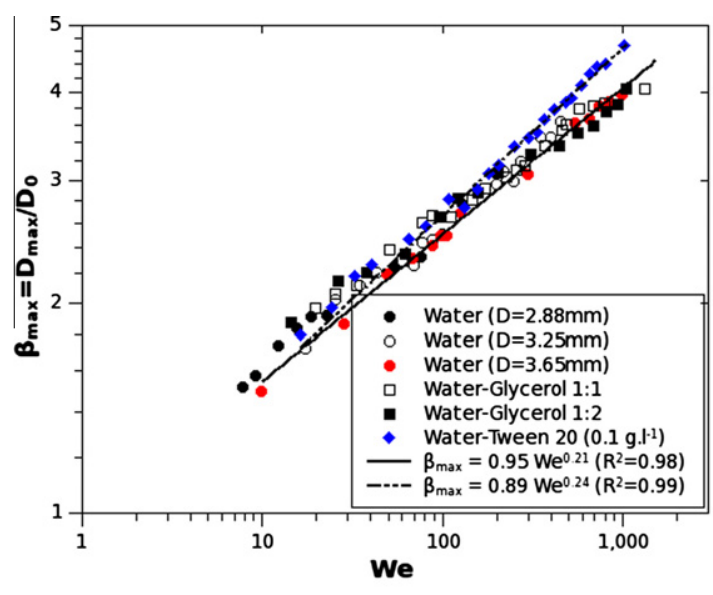

(A)

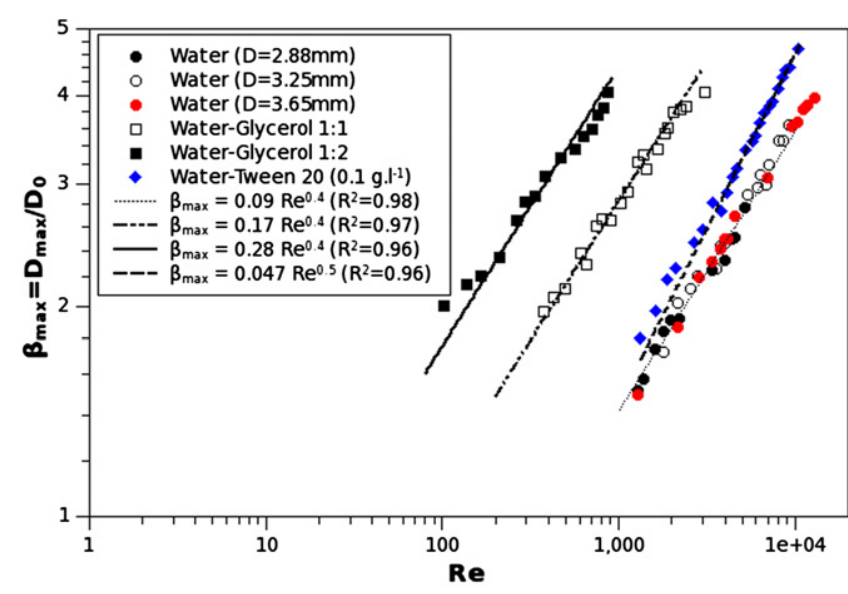

(B)

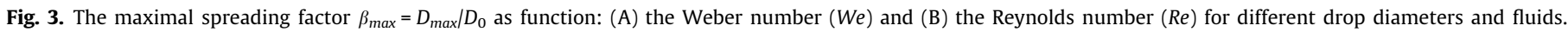
Equations of the best fit lines are included on the graph with their coefficients of determination, $R^{2}$.

Thus, $\beta_{\max }$ (water + Tween) scales with $W e^{0.24}$. Making the approximation that $W e^{0.24} \approx W e^{1 / 4}$, the maximum spreading factor for can be rewritten: $\beta_{\max }($ water + Tween $) \sim W e^{1 / 4}$. Finally, Fig. 3A shows that the role played by the surfactant on the drop behavior after impact was significant for high inertia, i.e. for $W e>200$.

On the other hand, when the viscosity of the drop was modified, the maximum spreading factor was well fitted by a straight line of slope $0.21 \pm 0.01$ (with a high coefficient of determination, $\left.R^{2}=0.98\right): \quad \beta_{\max }($ water $)$ or $\beta_{\max }($ water + glycerol $)$ scales with $W e^{0.21}$. Making the approximation that $W e^{0.21} \approx W e^{1 / 5}$, the maximum spreading factor can also be rewritten: $\beta_{\max }($ water $) \sim W e^{1 / 5}$ or $\beta_{\max }($ water + glycerol $) \sim W e^{1 / 5}$. As expected, the slope was smaller that in the previous case $\left(\beta_{\max }(\right.$ water + Tween $\left.) \sim W e^{1 / 4}\right)$ since the initial kinetic energy was much dissipated by the viscosity. The spreading was arrested by the viscous forces. We can notice that this scaling law, $\beta_{\max } \sim W e^{1 / 4}$, was similar to that found for the impact of a liquid drop of low viscosity on a super-hydrophobic surface [28].

In Fig. 3B, the maximum spread factor $\left(\beta_{\max }=D_{\max } / D_{0}\right)$ is plotted $v$ s. the Reynolds number. For water + Tween drops the maximum spreading factor $\beta_{\max }$ scales with $\operatorname{Re}^{1 / 2}$ while for the other liquid drops $\beta_{\max } \sim R e^{2 / 5}$. These results can be deduced of Fig. 3A. Indeed, in the case of a unique drop and even for drops of different diameters among values considered in this work $\left(\left(D_{01} / D_{02}\right)^{\frac{1}{4}} \in[1,1.1]\right)$, a law of the type $\beta_{\max } \sim W e^{\frac{1}{4}}$ leads to $\beta_{\max } \sim R e^{\frac{1}{2}}$ since $W e^{\frac{1}{4}} \sim D_{0}^{-\frac{1}{4}} R e^{\frac{1}{2}}$. In the same manner, $\beta_{\max } \sim W e^{\frac{1}{5}}$ leads to $\beta_{\max } \sim \operatorname{Re}^{\frac{2}{5}}$ for the other fluid. Therefore, the exponent may become only from the impact velocity dependence.

Our results do not concord with the models reported by Rein [29] and Eggers [30], for the drop impact on a solid surface. Indeed after impact on a solid surface, the initial kinetic energy $\left(E_{K} \sim \rho D_{0}^{3} V_{0}^{2}\right)$ is used to overcome the viscous resistance $\left(E_{\mu} \sim \mu V_{0} D_{\max }^{5} / D_{0}^{3}\right)$ and to provide the increase in surface energy $\left(E_{S} \sim \gamma D_{\max }^{2}(1-\cos \theta)\right)$ as the droplet spreads. Thus, for cases where the surface tension force plays a dominant role in arresting the drop spreading as compared to the viscous force, $E_{K} \sim E_{S}$ and $\beta_{\max } \sim W e^{1 / 2}$ whereas our experimental results of impact of drop on granular medium suggest that $\beta_{\max } \sim W e^{1 / 4}$. For cases in which the viscous forces are much more dominant in arresting the spreading $E_{K} \sim E_{\mu}$ and $\beta_{\max } \sim R e^{1 / 5}$. In this case our experimental results found $\beta_{\max } \sim R e^{2 / 5}$. This difference in scaling may be explained since the granular medium dissipate energy during the impact. Indeed, it is necessary to consider the energy to excavate the crater and elevate its initial mass (composed of beads) to a height at less equal to its depth. Moreover, the packing fraction is another parameter that could influence the energy dissipation. We excepted that an impact on a loose random packing bed will be more damped than an impact on a dense random packing bed. Furthermore, in order to predict more accurately the maximum spread factor of drop impact on solid surface various empirical correlations depending on Weber and Reynolds numbers were proposed: the Scheller and Bousfield [31] correlation, Roisman [32] correlation, etc. However, our experimental results have followed none of these correlations. Furthermore, an attempt to find a new relationship $\beta_{\max }=f(R e, W e)$ was failed. To explain this failure, we believe that the behavior of granular media (i.e. of the impact) is strongly influenced by the physical properties of the liquid drop.

Finally more recently, Katsuragi [6] reported that after the impact of a free-falling water drop $(W e<300)$ onto a granular layer, $\beta_{\text {max }}$ scales as $W e^{\frac{1}{4}}$ whereas we found $W e^{\frac{1}{5}}$. This difference between the results may be explained, as the liquid used is the same, by the fact that less energy was transmitted to the granular medium and dissipated into it during the drop deformation. Indeed, Katsuragi [6] used a very fine granular $(4-50 \mu \mathrm{m})$ with a packing fraction smaller than 0.50 (against 0.60 in our case). Another reason may be that the spreading process slightly depends on the capacity of absorption of the liquid by the granular bed.

\subsubsection{Splashing threshold}

In the case of drop impacts on solid surfaces or on thin liquid layers, transition between splashing and non-splashing has been studied in details [8]. However, to the best of our knowledge, splashing threshold of drop impact on granular medium was never reported. To delineate the boundary between splashing and non-splashing regions, the method reported by Mundo et al. [33] has been used. The method consists to report the drop impact behavior (splash or non-splash) as function of the splashing parameter $K_{d}\left(K_{d}=\right.$ $\left.W e^{1 / 2} \operatorname{Re}^{1 / 4}=\left(\frac{\rho^{3} D_{0}^{3} V_{0}^{5}}{\mu \gamma^{2}}\right)^{1 / 4}\right)$ and the Reynolds number Re. Indeed, it is well-known that drop impacts correlated much better with the splashing parameter $K_{d}$ than with the only We number. In Fig. 4, the drop impact behavior is reported for various splashing parameters $K_{d}$ and Reynolds numbers Re. Each symbol shape correspond to particulate fluid. The filled symbols correspond to splashing drops 


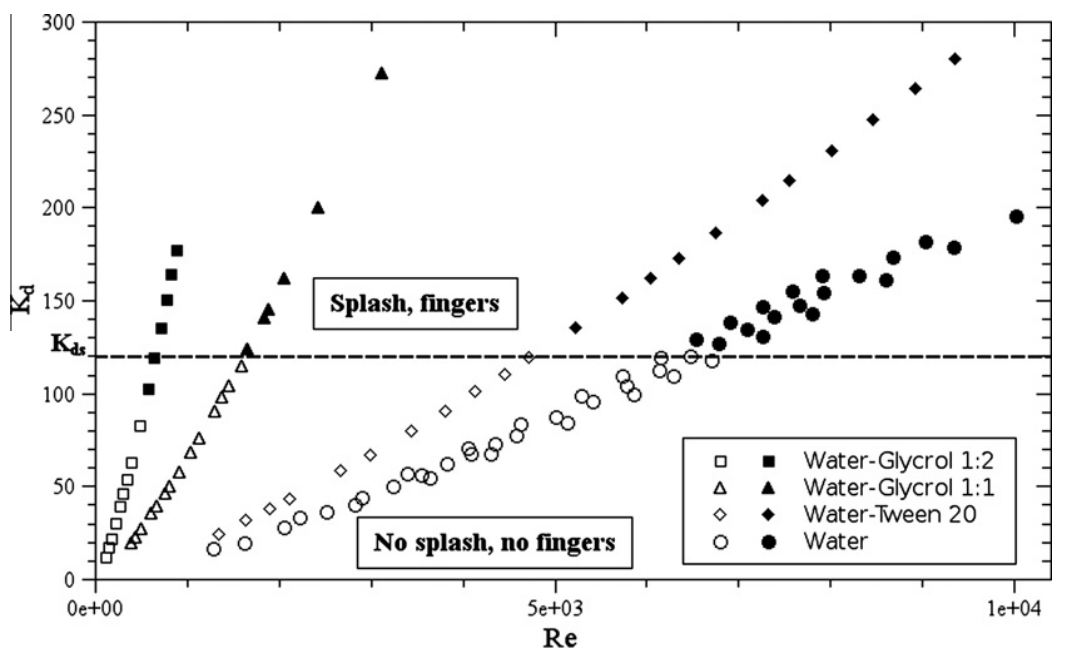

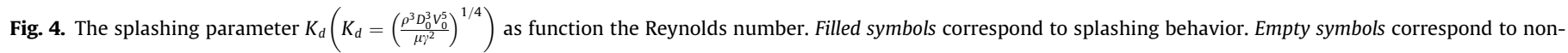
splashing behavior. The dotted line indicates the splashing threshold $\left(K_{d s}=120\right)$.

while the empty symbols correspond to impacting drops where non-splashing occurred. A clear boundary between these behavior regimes was found for a value of approximately $K_{d s}=120$. In comparison, the drop splashing threshold for a solid smooth impermeable surface corresponds to $K_{d s}=57.7$ [33] while for porous polyacrylonitrile nanofibers (with a pore diameter of the order of $5 \mu \mathrm{m}$ ) $K_{d s}=87$ [34]. Cossali et al. [35] established that the criterion for obtaining the splashing of a drop on thin liquid layer (with a thickness of $0.1 D$ ) is $K_{d s}=127$. The splashing parameter increase with the film thickness. These results indicate that the granular medium delayed the splashing. To splash, a drop (with the same diameter and physical properties) impacting on a granular medium needs higher velocity than that impacting upon a solid dry surface.

\subsection{Drop absorption}

From Fig. 1A and B, the absorption time ( $\left.t_{\text {measured }}\right)$ of two water drops with different drop sizes $\left(D_{0}\right)$ was measured. As reported in Table $2, t_{\text {measured }}=0.13 \mathrm{~s}$ for the drop with a small diameter $\left(D_{0}=2.88 \mathrm{~mm}, W e \sim 100\right)$ and $t_{\text {measured }}=0.09 \mathrm{~s}$ for the drop with a bigger diameter $\left(D_{0}=3.65 \mathrm{~mm}, W e \sim 650\right)$. It was surprising at first sight to find that the biggest drop took less time to be absorbed (an average bulk flow almost three times higher). However, from an inspection of the video data, it found that the absorption mechanisms were not the same in the two cases because the impacting drops developed different spreading behaviors.

In the first case in particular, and for all impacts at $W e / \sqrt{R e} \ll 1$ in general, the majority of drop absorption takes place after the drop spreading and receding and while the fluid is at rest. A fluid in a drop-like shape is then absorbed (see Fig. 1A the two last frames). Absorption and receding are quite independent and the latter is governed by capillary forces.

In the second case, absorption is observed to start relatively early and before the drop starts receding. This was observed for almost all impacts at $W e / \sqrt{R e} \gg 1$ where a flat film is absorbed instead of a spherical drop. In fact, the higher the impact kinetic energy, the bigger the drop maximal extension and the thinner the spreading film. Besides, the fluid film absorption does not start at the periphery, where the film thickness may be thought to be the lowest. Actually, the fluid film thickness decreases from the center to periphery at first spreading stages $[28,36]$. However, when the spreading lamella reaches its maximal extension, a blob appears at the rim. While there is no global fluid motion $\left(\frac{d \beta}{d t^{*}}=0\right.$ in
Table 2

Measured $\left(t_{\text {measured }}\right)$ and calculated $\left(t_{\text {calculated }}\right)$ absorption times for different drop sizes $\left(D_{0}\right)$, Weber number We and fluids. The fluid-granular medium contact angle, $\theta_{d}$, is also reported.

\begin{tabular}{llllll}
\hline Fluid & $\begin{array}{l}D_{0} \\
(\mathrm{~mm})\end{array}$ & $\theta_{d}\left(^{\circ}\right)$ & $W e$ & $\begin{array}{l}t_{\text {measured }} \\
(\mathrm{s})\end{array}$ & $\begin{array}{l}t_{\text {calculated }} \\
(\mathrm{s})\end{array}$ \\
\hline Water & 2.88 & $84 \pm 3$ & 150 & 0.13 & 0.3 \\
Water & 3.65 & $84 \pm 3$ & 750 & 0.09 & 0.5 \\
$\quad$ Water + Tween $(\approx 1$ & 3 & $79 \pm 3$ & 164 & 0.27 & 0.6 \\
$\quad$ CMC) & & & & & \\
Water + Tween $(\approx 1$ & 3 & $79 \pm 3$ & 549 & 0.05 & 0.65 \\
$\quad$ CMC) & & & & & \\
Water-glycerol 1:1 & 3.47 & $83 \pm 3$ & 114 & 1.68 & 4 \\
Water-glycerol 1:1 & 3.47 & $83 \pm 3$ & 570 & 1.06 & 4 \\
Water-glycerol 1:2 & 3.39 & $83 \pm 3$ & 119 & 2.29 & 12.5 \\
Water-glycerol 1:2 & 3.39 & $83 \pm 3$ & 546 & 2.43 & 12.5 \\
\hline
\end{tabular}

Fig. 2), a local flow from the center to the periphery still exists, feeding the blob and thinning the fluid film at its center [36]. Fluid central sucking induces lamella receding. Fig. 1B for $t=11.2 \mathrm{~ms}$ clearly shows the presence of fluid in the impact center. Therefore absorption is not the last stage of drop deformation. Absorption and receding are tightly coupled and we talk about an absorption induced by receding.

Table 2 reports measured absorption times $\left(t_{\text {measured }}\right)$ for different We and fluids from an inspection of the video data. Some interesting remarks can be made. First, these figures confirm that absorption times are systematically (except for very viscous fluids) higher for low We as explained above. Second, when the fluid viscosity increases, the absorption time was less depend on We (see Fig. 1C). Indeed, when We was changed, for glycerol-water 1:2 (19 times more viscous than water) $t_{\text {measured }}$ was varied of about $5 \%$ whereas the absorption times varied of about $45 \%$ and $450 \%$ for water or water-Tween mixture, respectively.

It may be interesting to calculate the theoretical absorption times and to compare them with experimental results. The method presented and confirmed in some particular cases by Hapgood et al. [5] was adopted. In Hapgood et al. [5] experiments, the drop was deposited with a syringe on the surface of the granular medium with no kinetic energy. With this method, the drop absorption was assumed to take place over a constant drop-granular medium contact surface (constant drawing area). Absorption time is then given by [5]: 
$t_{\text {calculated }}=1.35 \frac{V_{d}^{\frac{2}{3}}}{\epsilon^{2} R_{p}} \frac{\mu}{\gamma \cos \left(\theta_{d}\right)}$

where $V_{d}$ is the initial drop volume, $\epsilon$ is the porous medium porosity and $\theta_{d}$ the measured fluid-granular medium contact angle. $R_{p}$ is the measured mean pore radius using the Eq. (3), $R_{p}=12 \pm 1 \mu \mathrm{m}$. Thus, the drop size was at least 125 times greater than the pore size.

In our case, calculated absorption times approximated experimental times when experimental conditions very close to those of Hapgood et al. [5] i.e. for drop impacts with low We (see Table 2 ). For high We or high viscous fluids the absorption times calculated by the relationship (4) were failed because the model implies the implicit assumption that no absorption occurs during spreading and receding which is obviously not true. A more sophisticated model, as that presented by Lembach et al. [34] where it is shown that the absorption of drop impact on permeable media is different the Washburn's approach could be considered.

\subsection{Crater morphologies}

In Fig. 5, for different We, typical examples of crater morphologies observed by impacting into a container of glass beads a $3.65 \mathrm{~mm}$ diameter water drop are shown. The granular medium keeps a memory of the fluid shape at its maximal extension, thanks to its deformability and wettability. Similar craters were also observed with the different fluids. At low We, e.g. We $\approx 30$ (Fig. 5 crater with in its center a circular agglomeration of glass beads was formed. A rotationally symmetric rim was raised above the original target surface level. Glass beads driven by the receding drop formed a central agglomeration where the surface energy is sufficiently strong to join glass beads by capillary cohesion forces. This crater morphology was observed for We $\leqslant 100$. As We was increased the first irregularities appeared inside the crater and the agglomeration in the center was less circular (Fig. 5B). These irregularities became more marked at higher We (Fig. 5C and D). They can be associated with the fingering instability at the rim of the expanding water drop like reported in the previous studies of the water drop on a solid surface $[37,38]$. This crater morphology occurred in the range $100 \leqslant W e \leqslant 300$. This kind of crater was not reported by Katsuragi [6]. In its work, finer grains were used and lower We numbers were considered which may explain these differences. At higher kinetic energy, a layer of wet glass beads appears instead of the central agglomeration previously described (Fig. 5E). It is due to the water drops splashing.

A surprising and striking phenomenon was also observed, from the observation of Fig. 5, the crater depth did not increase with impact kinetic energy even if more energy was transmitted to the granular medium. For a solid impacting on granular medium with small kinetic energy to form a crater, the depth $d$, follows the relationship [39]: $\frac{d}{d_{0}}=\left(\frac{H}{d_{0}}\right)^{1 / 3}$, where $d_{0}$ is the minimum penetration for $h=0$ and $H=h+d$ the total drop distance. Thus, the crater depth increases with the kinetic energy of the impact.

In Fig. 6 , the crater rim diameter $D_{\text {rim }}$, measured at the top of the crater rim, is plotted against kinetic energy of drops at impact $\left(E_{K}=\frac{\pi}{6} \rho D_{0}^{3} g h\right)$. As expected, for different drop diameters and fluids, the measured diameter $D_{\text {rim }}$ increased with increasing $E_{K}$ on more than three orders of magnitude. The crater diameter $D_{\text {rim }}$ was different from maximal drop extension diameter $D_{m} a x$ since beads are ejected after the impact as reported by Nefzaoui et al. [7]. For water + Tween drops, the data was well fitted by a straight line of slope 0.18 (with a high coefficient of determination, $R^{2}=0.99$ ) therefore $D_{\text {rim }} \sim E_{K}^{0.18}$. Whilst for the other fluids, the data was well fitted by a straight line of slope $0.22 \pm 0.01$ (with a high coefficient of determination, $\left.R^{2}=0.96\right): D_{\text {rim }} \sim E_{K}^{0.22}$. These law

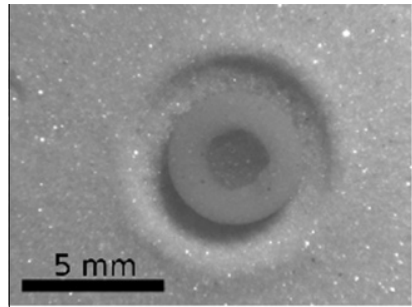

(A) $W e \approx 30$

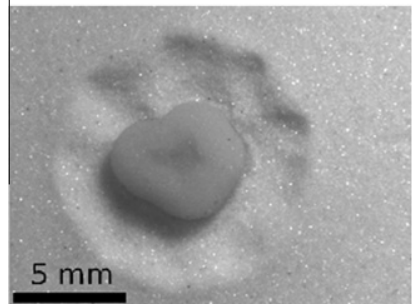

(C) $W e \approx 200$

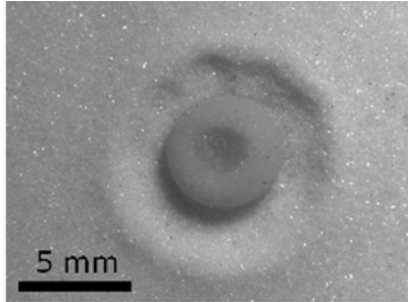

(B) $W e \approx 130$

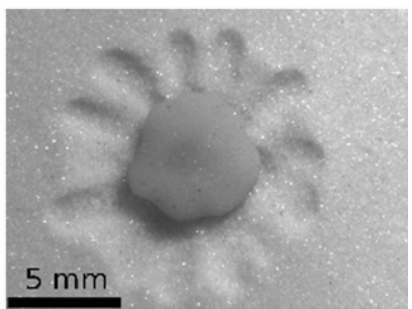

(D) $W e \approx 280$

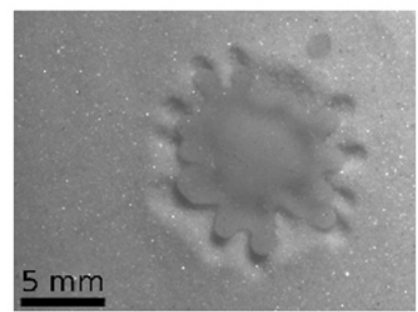

(E) $W e \approx 380$

Fig. 5. Crater morphologies observed by dropping a $3.65 \mathrm{~mm}$ diameter water drop into a container of glass beads for different Weber numbers.

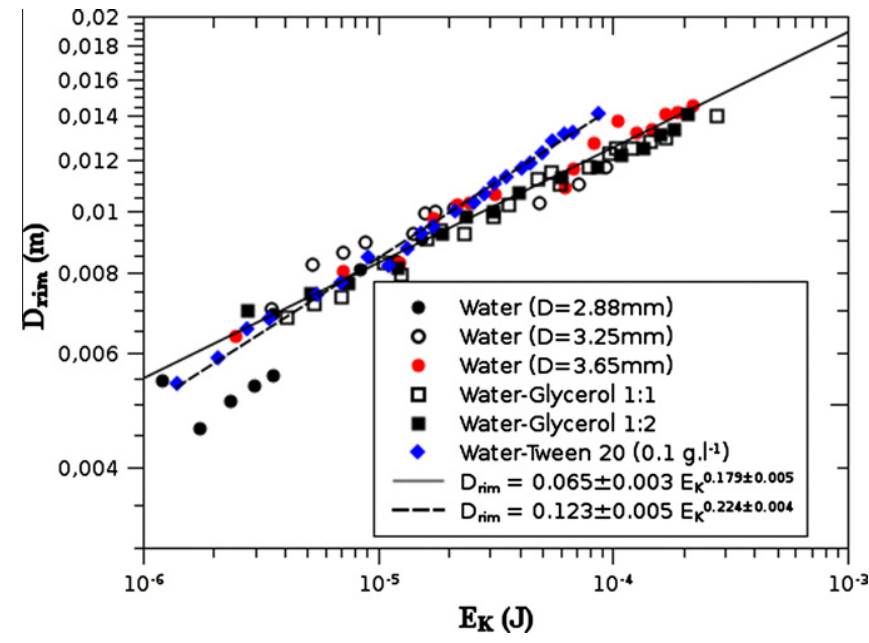

Fig. 6. Crater rim diameter $\left(D_{\text {rim }}\right)$ as function of the kinetic energy $\left(E_{K}\right)$ for different drop diameters and fluids. Equations of the best fit lines are included on the graph.

were slightly different of those reported for the solid projectile impact where $D_{\text {rim }}$ was reported to scale as $E_{K}^{\frac{1}{4}}[16,40,6]$. In fact, the process involved in the drop impact is very different from that involved in crater formation by the impact of a solid sphere into a granular medium. In particular, the crater generated by the drop impact is essentially formed by the drop pushing the surface grains outwards as it deforms. The fact that the crater formation only involved the grains nearest the surface seems to be the reason why the crater depth was not depend on kinetic energy of impact. 


\section{Conclusion}

An experimental study of liquid drop impacts on a granular medium has been presented. The drop free fall height was varied to obtain a Weber number (We) between 10 and 2000 and a Reynolds number $(R e)$ between 100 and 13000 . Four liquids were used to vary either the viscosity (between 1 and $19 \mathrm{mPa}$ ) or the surface tension (between 36 and $72 \mathrm{~mJ} \mathrm{~m}^{-2}$ ). The temporal evolution of the spreading factor $\left(\beta(t)=D(t) / D_{0}\right)$, spreading receding was detailed. The role plays by the surfactant on the drop behavior after impact was found significant especially when the kinetic energy was high. On the other hand, the final diameter of the drop was found insensitive to the viscosity of the fluid and the Weber number. The maximum spreading factor was determined. For water + Tween drops, i.e. for low surface tension, $\beta_{\max } \propto W e^{\frac{1}{4}}$ whereas, for the other fluids, i.e. with higher surface tension but various viscosities, $\beta_{\max } \propto W e^{\frac{1}{5}}$. An attempt to find a new relationship $\beta_{\max }=f(R e, W e)$ was failed. Thus, we believe that the behavior of granular media (i.e. of the impact) is strongly influenced by the physical properties of the liquid drop.

The boundary between splashing and non-splashing was determined using the splash parameter $K_{d}=W e^{1 / 2} R^{1 / 4}$. The threshold value, $K_{d s}=120$ was found higher that for impact of liquid drops on solid dry substrates $\left(K_{d}=57.7\right)$ but smaller than the splashing of a drop on thin liquid layer $\left(K_{d s}=127\right)$. These results indicate that the granular medium delayed the splashing; a drop impacting on a granular medium needs higher velocity than that impacting upon a solid dry surface to splash.

Measured absorption times depended highly on We and the fluid viscosity. Calculated times according to the simplified model reported by Hapgood et al. [5] were not in agreement with experiments. A better prediction of absorption times must be achieved taking into account absorption during spreading and receding or using a more complex model like that of Lembach et al. [34].

The morphology of the craters was discussed. In particular, it was reported that the crater depth did not increase with impact kinetic energy even if more energy was transmitted to the granular medium. The crater diameter, $D_{\text {rim }}$ was found to scale with $E_{K}^{0.18}$ for water + Tween drops and $E_{K}^{0.22}$ for the other fluids.

Finally, more experiments are necessary to understand the fluid granular interactions and to determine the influence of the granular size on the drop impact behavior. Furthermore, it will be interesting to develop an energetic model that details the initial drop kinetic energy dissipation during the drop deformation (viscous dissipation, etc.) and the crater formation (granular dissipation).

\section{Acknowledgement}

The authors thank F. Melo for discussions and help.

\section{References}

[1] V. Vaclavik, E. Christian, Essentials of Food Science, third ed., Springer, 2008

[2] A.I.J.M. van Dijk, L.A. Bruijnzeel, C.J. Rosewell, Rainfall intensity-kinetic energy relationships: a critical literature appraisal, J. Hydrol. (Amsterdam Netherlands) 261 (2002) 1

[3] J. Ries, M. Seeger, T. Iserloh, S. Wistorf, W. Fister, Calibration of simulated rainfall characteristics for the study of soil erosion on agricultural land, Soil Tillage Res. 106 (2009) 109.

[4] K. Holman, M. Cima, S. Uhland, E. Sachs, Spreading and infiltration of inkjetprinted polymer solution droplets on a porous substrate, J. Colloid Interface Sci. 249 (2002) 432.

[5] K. Hapgood, J. Litster, S. Biggs, T. Howes, Drop penetration into porous powder beds, J. Colloid Interface Sci. 253 (2002) 353.
[6] H. Katsuragi, Morphology scaling of drop impact onto a granular layer, Phys Rev. Lett. 104 (2010) 218001.

[7] E. Nefzaoui, O. Skurtys, Water drop impact on granular medium, in: Book of Abstracts, Southern Workshop on Granular Materials - SWGM09, 2009.

[8] A. Yarin, Drop impact dynamics: splashing, spreading, receding, bouncing ..., Annu. Rev. Fluid Mech. 38 (2006) 159.

[9] R. Rioboo, M. Marengo, C. Tropea, Time evolution of liquid drop impact onto solid, dry surfaces, Exp. Fluids 33 (2002) 112

[10] R.V. Wal, G. Berger, S. Mozes, The splash/non-splash boundary upon a dry surface and thin fluid film, Exp. Fluids 40 (2006) 53.

[11] M. Wang, Y. Hung, F. Lin, S. Lin, Dynamic behaviors of droplet impact and spreading: a universal relationship study of dimensionless wetting diameter and droplet height, Exp. Therm. Fluid Sci. 33 (2009) 1112-1118.

[12] R. Kannan, D. Sivakumar, Impact of liquid drops on a rough surface comprising microgrooves, Exp. Fluids 44 (2008) 927.

[13] L. Xu, Liquid drop splashing on smooth, rough, and textured surfaces, Phys. Rev. E 75 (2007) 056316.

[14] E. Nelson, H. Katsuragi, P. Mayor, D. Durian, Projectile interactions in granular impact cratering, Phys. Rev. Lett. 101 (2008) 068001.

[15] G. Caballero, R. Bergmann, D.V. der Meer, A. Prosperetti, D. Lohse, Role of air in granular jet formation, Phys. Rev. Lett. 99 (2007) 018001.

16] A. Walsh, K. Holloway, P. Habdas, J. de Bruyn, Morphology and scaling of impact craters in granular media, Phys. Rev. Lett. 91 (2003) 104301.

[17] X. Zheng, Z. Wang, Z. Qiu, Impact craters in loose granular media, Eur. Phys. J E: Soft Matter Biol. Phys. 13 (2004) 321.

[18] D. Lohse, R. Bergmann, R. Mikkelsen, C. Zeilstra, D.V. der Meer, M. Versluis, K.V. der Weele, M.V. der Hoef, H. kuipers kuipers, Impact on soft sand: void collapse and jet formation, Phys. Rev. Lett. 93 (2004) 198003.

[19] D. Beladjine, M. Amni, A. Valance, L. Oger, Collision process between an incident bead and a three-dimensional granular packing, Phys. Rev. E 75 (2007) 061305.

[20] W. Wakeham, M. Assael, A. Marmur, J. De-Coninck, T. Blake, S. Theron, E. Zussman, Handbook of Experimental Fluid Mechanics, Springer-Verlag, 2007. pp. 96-105.

[21] A. Biance, F. Chevy, C. Clanet, G. Lagubeau, D. Quere, On the elasticity of an inertial liquid shock, J. Fluid Mech. 554 (2006) 47-66.

[22] K. Rao, P. Nott, An Introduction to Granular Flows, Cambridge University Press, 2008.

[23] R. Nedderman, Statics and Kinematics of Granular Materials, Cambridge University Press, 1992

[24] H. Xue, Z. Fang, Y. Yang, J. Huang, L. Zhou, Contact angle determined by spontaneous dynamic capillary rises with hydrostatic effects: experiment and theory, Chem. Phys. Lett. 432 (2006) 326.

[25] A. Clarke, T. Blake, K. Carruthers, A. Woodward, Spreading and imbibition of liquid droplets on porous surfaces, Langmuir 18 (2002) 2080-2084.

[26] X. Zhang, O. Basaran, Dynamic surface tension effects in impact of a drop with a solid surface, J. Colloid Interface Sci. 187 (1997) 166-178.

[27] S. Sikalo, M. Marengo, C. Tropea, E.N. Ganic, Analysis of impact of droplets on horizontal surfaces, Exp. Therm. Fluid Sci. 25 (2002) 503.

[28] C. Clanet, C. Beguin, D. Richard, D. Quere, Maximal deformation of an impacting drop, J. Fluid Mech. 517 (2004) 199.

[29] M. Rein, The transitional regime between coalescing and splashing drops, J. Fluid Mech. 306 (1996) 145.

[30] J. Eggers, M.A. Fontelos, C. Josserand, S. Zaleski, Drop dynamics after impact on solid wall: theory and simulations, Phys. Fluids 22 (2010) 062101.

[31] B.L. Scheller, D.W. Bousfield, Newtonian drop impact with a solid surface, AICHE J. 41 (1995) 1357-1367.

[32] I. Roisman, Inertia dominated drop collisions. II. An analytical solution of the Navier-Stokes equations for a spreading viscous film, Phys. Fluids 21 (2009) 10.

[33] C.H.R. Mundo, M. Sommerfeld, C. Tropea, Droplet-wall collision: experimenta studies of the deformation and breakup process, Int. J. Multiphase Flow 21 (1995) 151-173.

[34] A. Lembach, H. Tan, I. Roisman, T. Gambaryan-Roisman, Y. Zhang, C. Tropea, A Yarin, Drop impact, spreading, splashing, and penetration into electrospun nanofiber mats, Langmuir 26 (2010) 9516-9523.

[35] G.E. Cossali, A. Coghe, M. Marengo, The impact of a single drop on a wetted solid surface, Exp. Fluids 22 (1997) 463.

[36] I.V. Roisman, R. Rioboo, C. Tropea, Normal impact of a liquid drop on a dry surface: model for spreading and receding, Proc. Roy. Soc. London, Ser., A 458 (2002) 1411

[37] R. Allen, The role of surface tension in splashing, J. Colloid Interface Sci. 51 (1975) 350

[38] N. Mehdizadeh, S. Chandra, J. Mostaghimi, Formation of fingers around the edges of a drop hitting a metal plate with high velocity, J. Fluid Mech. 510 (2004) 353.

39] M.A. Ambroso, R.D. Kamien, D.J. Durian, Dynamics of shallow impact cratering, Phys. Rev. E 72 (2005) 041305.

[40] J. Uehara, M. Ambroso, R. Ojha, D. Durian, Low-speed impact craters in loose granular media, Phys. Rev. Lett. 90 (2003) 194301. 